HUTP-95/A003

hep-th/9501131

\title{
Some Three Generation (0,2) Calabi-Yau Models
}

\author{
Shamit Kachru ${ }^{\dagger}$ \\ Lyman Laboratory of Physics \\ Harvard University \\ Cambridge, MA 02138
}

\begin{abstract}
It has recently been realized that a large class of Calabi-Yau models in which the VEV of the gauge connection is not set equal to the spin connection of the Calabi-Yau manifold are valid classical solutions of string theory. We provide some examples of three generation models based on such generalized Calabi-Yau compactifications, including models with observable gauge group $S U(3) \times S U(2) \times U(1)$.
\end{abstract}

January 1995

$\dagger$ Junior Fellow, Harvard Society of Fellows 


\section{Introduction}

String theory is the leading (and at present, only) candidate for a unified theory of the fundamental interactions. However, daunting challenges remain to be overcome, both in our fundamental understanding of strings and in connecting string theory with low energy physics.

Although at present we cannot make definite statements about generic properties of string-based phenomenology, one of the obvious things we can do is construct examples of pseudo-realistic string compactifications and explore, with judicious assumptions, the resulting phenomenology. The purpose of such explorations is of course not to find the model of nature, but to see if one can find generic advantages or deficiencies of stringderived models.

To that end, in this paper we provide several examples of three-generation string compactifications on Calabi-Yau manifolds [1]. While the Calabi-Yau models most commonly studied to date have been $(2,2)$ supersymmetric models which naturally yield an $E_{6}$ effective GUT group, $(0,2)$ Calabi-Yau models provide a much broader class of compactifications in which one can also naturally obtain $S O(10)$ or $S U(5)$ as the effective gauge group [2,3]. It has recently been proved that, contrary to previous expectations [4, many $(0,2)$ Calabi-Yau models are true solutions of string theory (both non-perturbatively in the sigma model expansion and to all orders in the string loop expansion) [5,6]. It therefore seems worthwhile to explore the enhanced phenomenological opportunities in this large new class of classical string vacua, and we take a first step in that direction in this paper.

In $\S 2$, we briefly describe the class of models under consideration [7]. In $\S 3$, we provide four examples in this class of models which yield three generations of chiral fermions in the normal unification representations of $E_{6}$ and $S U(5)$. These models are not related to the $(2,2)$ theories on the same Calabi-Yau manifolds by holomorphic deformation. Two of the examples, with gauge group $S U(5)$, are on manifolds with nontrivial $\pi_{1}$. In $\S 4$, using Wilson lines as a tool for gauge symmetry breaking [8,9], we break the gauge group in these two $S U(5)$ examples to $S U(3) \times S U(2) \times U(1)$. It will be clear that our results are in no sense exhaustive - one could certainly construct many other models along similar lines.

To our best knowledge, only one three-generation Calabi-Yau model has been explored in any depth in the literature - see [10,11, 12, 13, 14] and references therein. Several three

1 The conclusions of the forthcoming paper [6] in particular are stronger than those of [5] and imply that all of the models considered here are bona fide solutions of string theory. 
generation $(2,2)$ models have been listed in [15] and [16] (the latter reference classifies all CY hypersurfaces in weighted projective four-spaces), all of which are compactifications on manifolds with trivial $\pi_{1}$. 2 Considerably more work has been directed towards exploring semi-realistic string models based on toroidal orbifolds [17] and free-fermions [18]. It may prove interesting to investigate the phenomenology of the models presented here, or other similar models; we hope to undertake such investigations in the near future.

\section{A Class of $(0,2)$ Calabi-Yau Models}

Recall that the data which enters in specifying a $(0,2)$ Calabi-Yau model is a choice of Calabi-Yau manifold $M$ and stable, holomorphic vector bundles $V_{1}$ and $V_{2}$ (representing the vacuum configurations of the gauge fields in the observable and hidden $E_{8}$ of the heterotic string) satisfying

$$
\begin{gathered}
c_{2}(M)=c_{2}\left(V_{1}\right)+c_{2}\left(V_{2}\right) \\
c_{1}\left(V_{1,2}\right)=0 \bmod 2 .
\end{gathered}
$$

Here the $c_{i}$ are the Chern classes of the vector bundles in question. (2.1) is the well known anomaly cancellation condition, while (2.2) is the requirement that $V_{1}$ and $V_{2}$ admit spinors.

In addition to these topological conditions, there are perturbative conditions for conformal invariance of a $(0,2)$ Calabi-Yau $\sigma$-model [2]. At lowest order, we must require that the metric on $M$ be the familiar Ricci-flat Kahler metric $g_{i \bar{j}}$ whose existence is guaranteed by Yau's theorem. In addition, the connections on $V_{1,2}$ must satisfy

$$
g^{i \bar{j}} F_{i \bar{j}}=0 .
$$

For stable bundles $V_{1,2}$, a theorem of Uhlenbeck and Yau guarantees the existence of a solution to (2.3) as long as the integrability condition

$$
\int_{M} J \wedge J \wedge c_{1}\left(V_{1,2}\right)=0
$$

is met, where $J$ is the Kahler form of $M$. We will satisfy (2.4) by choosing $c_{1}\left(V_{1,2}\right)=0$ (as indeed we must if we work on a manifold $M$ with $h_{1,1}=1$ ). Higher orders of sigma model perturbation theory do not lead to any further conditions on $M$ or $V_{1,2}$.

2 I am informed by A. Klemm that it is extremely difficult if not impossible to find examples of three generation models with nontrivial $\pi_{1}$ by orbifolding higher generation models in this class; this has been investigated by A. Niemeyer. 
We will confine our attention to non-singular Calabi-Yau manifolds $M$, defined by the vanishing loci of $N$ equations of degree $d_{i}(1 \leq i \leq N)$ in some $W C P_{w_{1} \ldots w_{N+4}}^{N+3}$ with homogeneous coordinates $\phi_{j}, 1 \leq j \leq N+4$. In such a model $M$ has only one harmonic $(1,1)$ form $J$ (it inherits the Kahler class of the ambient projective space), and one finds that

$$
\int_{M} J \wedge J \wedge J=\frac{\Pi d_{i}}{\Pi w_{j}} .
$$

This will be useful momentarily.

In the models of interest with $V_{1}$ of rank 3,4 , or 5 (spacetime gauge group $E_{6}$, $S O(10)$, or $S U(5)$ ), the net number of generations (\# generations - \# antigenerations) $N_{\text {gen }}$ of chiral fermions in the observable sector (in the $\mathbf{2 7}$ of $E_{6}, \mathbf{1 6}$ of $S O(10)$ or $\mathbf{1 0}+\overline{\mathbf{5}}$ of $S U(5))$ is given by

$$
N_{\text {gen }}=\frac{1}{2}\left|\int_{M} c_{3}\left(V_{1}\right)\right|
$$

(2.6) reflects the fact that massless fermions in four dimensions correspond to zero modes of the Dirac operator on the compactification manifold $M$, and follows from the index theorem. It reduces to the familiar expression $\frac{1}{2}|\chi|$ in the $(2,2)$ case. To determine separately the number of generations and the number of anti-generations instead of just the difference, one must also compute the dimension of $H^{1}\left(M, V_{1}\right)$.

Following [7], we consider vacuum gauge bundles which are defined by the following exact sequence:

$$
0 \rightarrow V \rightarrow \bigoplus_{a=1}^{r+M} \mathcal{O}\left(n_{a}\right) \stackrel{\otimes F_{a}^{i}(\phi)}{\longrightarrow} \bigoplus_{i=1}^{M} \mathcal{O}\left(m_{i}\right) \rightarrow 0 .
$$

Here $r=3,4,5$ yields gauge group $E_{6}, S O(10)$ or $S U(5)$, and the $n_{a}$ and $m_{i}$ are positive integers with $\sum m_{i}=\sum n_{a}$ (guaranteeing that $c_{1}(V)=0$ ). $\mathcal{O}(a)$ denotes the ath power of the hyperplane bundle of the ambient weighted projective space, and the $F_{a}^{i}(\phi)$ are polynomials homogeneous of degree $m_{i}-n_{a}$ in the $\phi$ s which never simultaneously vanish on $M$. For shorthand, we use the notation $V=\left(\left\{m_{i}\right\} ;\left\{n_{a}\right\}\right)$. The worldsheet quantum field theory which describes this spacetime model can be obtained as the infrared limit of a linear sigma model with $U(1)$ gauge group (for more details the reader should consult [7]) and the linear sigma model will contain $r+M$ left-moving fermions $\lambda_{1}, \cdots, \lambda_{r+M}$ with gauge charges $\left(n_{1}, \cdots, n_{r+M}\right)$.

3 Formulas such as this and other basic wisdom concerning Calabi-Yau spaces can be found in [19]. 
In general we will have nontrivial factors embedded in both the observable and hidden $E_{8} \mathrm{~s}$, and we will denote them by $V_{1}$ and $V_{2}$. For a theory with $V_{1}$ described as in (2.7), one finds that

$$
c_{3}\left(V_{1}\right)=-\frac{1}{3}\left(\sum_{i} m_{i}^{3}-\sum_{a} n_{a}^{3}\right) J^{3} .
$$

Combining (2.8) with (2.6) and (2.5), we see that determining the net number of generations of chiral fermions in such a compactification is a simple exercise in arithmetic. $H^{1}\left(M, V_{1}\right)$ also has a convenient deformation theoretic representation as M-tuples $\left(P_{1}(\phi), \cdots, P_{M}(\phi)\right)$ modulo $\left\{\left(F_{a}^{1}(\phi), \cdots, F_{a}^{M}(\phi)\right)\right\}$ where of course $P_{i}$ has degree $m_{i}$. This allows us to compute $\operatorname{dim} H^{1}\left(M, V_{1}\right)$ in the examples of $\S 3$ and verify that not only is (2.6) equal to three but there are in fact precisely three generations and no antigenerations.

\section{Some Three Generation Compactifications}

We now use the apparatus of $\S 2$ to construct some three-generation models. We begin with two very simple examples of $E_{6}$ theories and then discuss two more involved examples, which both give rise to $S U(5)$ gauge group (and are on non-simply connected manifolds, which admit gauge symmetry breaking by Wilson lines). In the second $S U(5)$ example, the hidden sector $E_{8}$ remains unbroken. In $\S 4$, we break the gauge group of the two $S U(5)$ examples down to $S U(3) \times S U(2) \times U(1)$ by using Wilson lines.

\subsection{Example 1: An $E_{6}$ Model}

Consider the compactification on the Calabi-Yau hypersurface $M$ defined by the vanishing locus of a degree ten polynomial in $W C P_{1,1,1,2,5}^{4}$ with $V_{1}=(2,2,2 ; 1,1,1,1,1,1)$ and $V_{2}=(9 ; 1,1,2,2,3)$. One easily computes that $\int_{M} J^{3}=1$ for this manifold, so $\int_{M} c_{3}\left(V_{1}\right)=-6$. Hence, this model has a net of three generations in the observable sector. The effective GUT group is $E_{6}$, and the charged matter fields transform in the $\mathbf{2 7}$ of $E_{6}$. Using the ideas in $\S 2$ it is easy to verify that $\operatorname{dim} H^{1}\left(M, V_{1}\right)=3$ so we have three generations and no antigenerations. This is true in our other examples as well, so we will not repeat the statement on each occasion.

\subsection{Example 2: Another $E_{6}$ Model}

Now, we look at an example on a complete intersection manifold. Let $M$ be the intersection of the vanishing loci of two degree six equations in $W C P_{1,1,2,2,3,3}^{5}$. Let $V_{1}=$

$(2,2,2 ; 1,1,1,1,1,1)$ and $V_{2}=(7 ; 1,1,1,2,2)$. Then $\int_{M} c_{3}\left(V_{1}\right)=-6$, so once again we have a compactification with three generations in the $\mathbf{2 7}$ of $E_{6}$. 


\subsection{Example 3: An SU(5) Model}

Next, we find an $S U(5)$ theory on a manifold with $\pi_{1}=Z_{3}$. Start as in Example 2 , with $M$ being the complete intersection of two degree six polynomials $P_{1}$ and $P_{2}$ in $W C P_{1,1,2,2,3,3}^{5}$. Choose $V_{1}=(3,3,2 ; 1,1,1,1,1,1,1,1)$ and $V_{2}=(6,3 ; 1,1,1,2,2,2)$. One can easily check that this theory has 9 generations in the $\overline{\mathbf{5}}+\mathbf{1 0}$ of $S U(5)$ in the observable sector, so we want to orbifold by a freely acting $Z_{3}$ to obtain a three-generation model.

Consider the $Z_{3}$ action generated by $g$ which acts as follows

$$
g: \quad \phi_{1,3,5} \rightarrow \alpha \phi_{1,3,5}, \quad \phi_{2,4,6} \rightarrow \alpha^{2} \phi_{2,4,6}
$$

where $\alpha=e^{i \frac{2 \pi}{3}}$. Given these transformation laws, one can write down nonsingular choices of $P_{1}, P_{2}$ which admit (3.1) as a symmetry; choose such an $M$. Then not only is (3.1) a symmetry of this manifold, but for generic choices it acts freely on $M$.

It must also preserve the holomorphic three-form $\Omega$ on $M$ if the quotient is to be a solution of string theory. By the general argument presented on p.495 of [20], one knows that the holomorphic three-form of $M$ will always descend to the quotient of $M$ by a freely

acting symmetry. This follows from the fact that the arithmetic genus $\sum_{k=0}^{3}(-1)^{k} h^{0, k}$ of the quotient must vanish. Nonetheless, it is instructive to explicitly verify the invariance of $\Omega$ (as practice for the case of quotients by non-freely acting symmetries, for example). Following $\S 3$ of [21] we see that the three-form can be written

$$
\Omega=\oint \oint \frac{\epsilon_{i j k l m n} \phi_{i} d \phi_{j} \wedge d \phi_{k} \wedge d \phi_{l} \wedge d \phi_{m} \wedge d \phi_{n}}{P_{1} P_{2}}
$$

where the contour integrals are taken about the loci $P_{1}=0$ and $P_{2}=0$. Since the numerator of the integrand transforms with an $\alpha^{9}=1$ while the denominator $P_{1} P_{2}$ is invariant as well, $\Omega$ does descend to the $Z_{3}$ orbifold .

At this point we have seen that the $Z_{3}$ acts freely on $M$ and preserves the holomorphic three-form, so if we were studying a $(2,2)$ model we would be done. However, to take a quotient of a $(0,2)$ model by such a discrete symmetry group $G$, one must also check that $G$ lifts to an automorphism of the vacuum gauge bundle $V$. In addition one has to check the level-matching conditions of [22] which are necessary for modular invariance. These conditions are more or less automatically satisfied in $(2,2)$ models but not $(0,2)$ models.

A brief summary of these conditions is as follows: Suppose we wish to orbifold our $(0,2)$ model by a $Z_{N}$ and let $\beta=e^{\frac{2 \pi i}{N}}$. Assume the $\phi_{i}$ transform as $\beta^{r_{i}}$, the fermions $\lambda_{a}^{1}$ 
associated with $V_{1}$ transform as $\beta^{r_{a}}$ and the fermions $\lambda_{b}^{2}$ associated with $V_{2}$ transform as $\beta^{\tilde{r}_{b}}$. Then the conditions which must be satisfied for $N$ even are

$$
\begin{gathered}
\sum_{i}\left(r_{i}\right)^{2}=\sum_{a}\left(r_{a}\right)^{2}+\sum_{b}\left(\tilde{r}_{b}\right)^{2} \bmod 2 N \\
\sum_{i} r_{i}=\sum_{a} r_{a}=\sum_{b} \tilde{r}_{b}=0 \bmod 2
\end{gathered}
$$

For odd $N$ one gets only the analogue of (3.3), and it must hold mod $N$ instead of mod $2 N$. Note that these conditions are necessary for modular invariance but are only known to be sufficient in the case of free field theory (on the worldsheet). Indeed, in interacting $(0,2)$ theories there are indications that extra constraints may be needed to ensure consistency at the one-loop level [23]. We will have nothing more to say about this here, however.

Let us choose the extension of $g$ as follows. Let $g$ act on the eight fermions associated to $V_{1}$ as

$$
g \text { on } V_{1}: \quad 1,1,1, \alpha, \alpha, \alpha^{2}, \alpha^{2}, \alpha^{2} .
$$

Let $g$ act on the fermions of worldsheet $U(1)$ gauge charges $(1,1,1,2,2,2)$ associated with $V_{2}$ as

$$
g \text { on } V_{2}: \quad 1, \alpha, \alpha^{2}, 1, \alpha, \alpha^{2} .
$$

It is now easy to check that the level-matching condition is satisfied. Choose the $F_{a}^{i}$ involved in defining $V_{1}$ to insure that the combinations $\lambda_{a}^{1} F_{a}^{i}$ (no sum on $a$ ) are $g$ invariant (one can make such choices), and do the same for $V_{2}$. This restricted set of $F$ s represents the set for which the chosen $g$ action is indeed an automorphism of $V$.

Having fulfilled the various consistency conditions, we see that we have found a freely acting $Z_{3}$ orbifold of the 9 generation theory in $W C P_{1,1,2,2,3,3}^{5}$. The result is a model with three $\overline{\mathbf{5}}+\mathbf{1 0}$ s of $S U(5)$ as the charged observable sector matter content. In addition, the target manifold has a nontrivial fundamental group $\pi_{1}=Z_{3}$.

It is worth emphasizing that because we have obtained this model as the quotient of the 9 generation model by a freely acting $Z_{3}$, the massless states in the 3 generation theory simply correspond to the $Z_{3}$ invariant massless states in the 9 generation theory. The twisted sectors only contribute massive states. In the case of quotients by non-free group actions, one would in general have some massless states coming from twisted sectors as well. 


\subsection{Example 4: Another SU(5) Model}

The last example we provide is an $S U(5)$ theory on the $Z_{5} \times Z_{5}$ orbifold of the quintic first studied in [1]. That is, we choose for $M$ the Fermat quintic

$$
\sum_{i=1}^{5} \phi_{i}^{5}=0
$$

in $C P^{4}$. Instead of starting with the $(2,2)$ theory on this quintic, however, we begin with $V_{1}=(3,3,3,3,3 ; 1,1,1,1,1,2,2,2,2,2)$ and we leave the second $E_{8}$ unbroken. It is easy to check that $\int_{M} c_{3}\left(V_{1}\right)=-150$ so orbifolding by a freely acting $Z_{5} \times Z_{5}$ will yield a three generation $S U(5)$ theory.

The $Z_{5} \times Z_{5}$ symmetry group of (3.7) that we wish to orbifold by is generated by

$$
\begin{gathered}
g_{1}:\left(\phi_{1}, \phi_{2}, \phi_{3}, \phi_{4}, \phi_{5}\right) \rightarrow\left(\phi_{2}, \phi_{3}, \phi_{4}, \phi_{5}, \phi_{1}\right) \\
g_{2}: \phi_{i} \rightarrow \alpha^{i} \phi_{i},
\end{gathered}
$$

where $\alpha=e^{\frac{2 \pi i}{5}}$. As discussed in [1], this discrete symmetry group does act freely on (3.7) and hence preserves the holomorphic three-form.

Now, we need to assign transformation laws to the $\lambda$ s and choose the holomorphic structure of $V_{1}$ so that the symmetries (3.8) and (3.9) lift to automorphisms of $V_{1}$. Let us imagine we are orbifolding first by (3.8), then by (3.9). Denote by $\lambda_{1, \cdots, 5}$ the five leftmoving fermions of worldsheet $U(1)$ gauge charge one and by $\lambda_{6, \cdots, 10}$ the five of gauge charge two. Choose

$$
F_{1}=\left(\phi_{1}^{2}+\phi_{3} \phi_{4}, \phi_{1}^{2}, \phi_{1}^{2}, \phi_{1}^{2}, \phi_{1}^{2}\right)
$$

(the $i$ th component of $F_{1}$ above represents $F_{1}^{i}$ in (2.7)) and similarly let $F_{2}, \cdots, F_{5}$ be given by

$$
F_{2}=\left(\phi_{2}^{2}, \phi_{2}^{2}+\phi_{4} \phi_{5}, \phi_{2}^{2}, \phi_{2}^{2}, \phi_{2}^{2}\right), \cdots, F_{5}=\left(\phi_{5}^{2}, \phi_{5}^{2}, \phi_{5}^{2}, \phi_{5}^{2}, \phi_{5}^{2}+\phi_{2} \phi_{3}\right) .
$$

To complete the specification of the holomorphic structure of $V_{1}$, choose

$$
F_{6}=\left(\phi_{1}, 0,0,0,0\right), F_{7}=\left(0, \phi_{2}, 0,0,0\right), \cdots F_{10}=\left(0,0,0,0, \phi_{5}\right) .
$$

Note that with the choices made, the five $F^{i}$ are linearly independent.

To complete our assignment of transformation laws, assign the $\lambda$ s the following transformation properties under $g_{1}$ :

$$
g_{1}:\left(\lambda_{1}, \lambda_{2}, \lambda_{3}, \lambda_{4}, \lambda_{5}\right) \rightarrow\left(\lambda_{2}, \lambda_{3}, \lambda_{4}, \lambda_{5}, \lambda_{1}\right)
$$




$$
g_{1}:\left(\lambda_{6}, \lambda_{7}, \lambda_{8}, \lambda_{9}, \lambda_{10}\right) \rightarrow\left(\lambda_{7}, \lambda_{8}, \lambda_{9}, \lambda_{10}, \lambda_{6}\right)
$$

If we now recall (see e.g. [7]) that the $\lambda_{a}$ and the $F_{a}^{i}$ really enter the action of our worldsheet field theory in the combination $P_{i} \lambda_{a} F_{a}^{i}$ where the $P_{i}$ s are $(0,2)$ chiral superfields, then we see that to truly make the quantum field theory action invariant under $g_{1}$ we should also have $g_{1}$ act to permute the $P_{i}$ in the same way as the $\phi_{i}$. It is apparent that with this choice of the $F$ s and the action of $g_{1}$ on $V_{1}$, the $Z_{5}$ symmetry does indeed lift to an automorphism of $V_{1}$. Diagonalizing the action of $g_{1}$ on the $\phi \mathrm{s}, P \mathrm{~s}$ and $\lambda_{\mathrm{s}}$, one can verify that the quadratic level-matching condition (3.3) is satisfied by the eigenvalues of $g_{1}$.

Now that we have successfully taken the $g_{1}$ orbifold (and are down to a 15 generation model), we must consider how to lift the action of $g_{2}$ to $V_{1}$. It is clear from the choice of the $F$ s above that we must assign the $\lambda_{\mathrm{s}}$ the following transformation under $g_{2}$ to keep $\lambda_{a} F_{a}^{i}$ invariant:

$$
\begin{gathered}
g_{2} \text { on } \lambda_{1, \cdots 5}: \quad \alpha^{3}, \alpha, \alpha^{4}, \alpha^{2}, 1 \\
g_{2} \text { on } \lambda_{6, \cdots, 10}: \quad \alpha^{4}, \alpha^{3}, \alpha^{2}, \alpha, 1 .
\end{gathered}
$$

And again it is simple to check that (3.3) is satisfied.

So finally, with the data specified above, the full $Z_{5} \times Z_{5}$ symmetry of the Fermat quintic lifts to an automorphism of $V_{1}$. Taking the $Z_{5} \times Z_{5}$ orbifold, we obtain a three generation $S U(5)$ theory, on a target manifold with $\pi_{1}=Z_{5} \times Z_{5}$.

\section{4. $\mathrm{SU}(5) \rightarrow \mathrm{SU}(3) \times \mathrm{SU}(2) \times \mathrm{U}(1)$}

In compactification on a manifold $M$ with $\pi_{1}(M) \neq 0$, one is allowed to give expectation values to Wilson lines around the noncontractable loops $\gamma$ in $M$

$$
U_{\gamma}=\mathrm{P} \exp \left(\oint_{\gamma} A d x\right)
$$

This amounts to a choice of a homomorphism from $\pi_{1}(M) \rightarrow G$, where $G$ is the spacetime gauge group. One is left with a vacuum with $G$ broken to the subgroup of $G$ which commutes with $U_{\gamma}$.

We will be choosing Wilson lines in some $Z_{N}$ subgroup of the spacetime gauge group (corresponding to a homomorphism mapping a $Z_{N}$ subgroup of $\pi_{1}(M)$ to $G$ ). One can think of the Wilson lines as acting on the gauge degrees of freedom of the heterotic string. We are interested in using Wilson lines in the $S U(5)$ theories of $\S 3$, so we take the relevant 
worldsheet gauge degrees of freedom to be four free left-moving bosons $X^{I}$. Turning on Wilson lines means that we should include sectors in which the $X^{I}$ (which live on a leftmoving torus) only close up to

$$
X^{I}(\sigma+\pi)=X^{I}(\sigma)+2 \pi \delta^{I}
$$

for some $\delta^{I}$. According to [24,25] we should impose the following analogue of the levelmatching constraint (3.3) on this orbifolding associated with the Wilson lines, if we are to obtain a modular invariant theory.

As in [9], choose

$$
U=\exp \left(2 \pi i \sum_{i=1}^{4} \delta^{i} H^{i}\right)
$$

where the $H^{i}$ generate the Cartan subalgebra of $S U(5)$. Consider $\left(\delta^{1}, \cdots, \delta^{4}\right)$ as a fourvector in the dual basis. Then the level-matching condition for Wilson lines is

$$
\frac{1}{2}(\delta, \delta)=0 \bmod \frac{1}{N}
$$

where the inner product $(\delta, \delta)=\delta_{i} A_{i j} \delta_{j}$ should be taken with an insertion of the $A_{4}$ Cartan matrix $A_{i j}$.

Following p. 71 of [26], we see that the unique choice (up to scale) of $\delta$ which breaks $S U(5)$ to $S U(3) \times S U(2) \times U(1)$ (and corresponds to choosing a Wilson line in the weak hypercharge $U(1))$ is given by

$$
\delta \sim(-2,1,-1,2)
$$

So we need to find such Wilson lines which correspond to $Z_{3}$ transformations (for the case of Example 3) and $Z_{5}$ transformations (for the case of Example 4) to accomplish the desired symmetry breaking.

In Example 3, with $\pi_{1}(M)=Z_{3}$, take

$$
\delta=\frac{1}{3}(-2,1,-1,2) .
$$

Some simple arithmetic tells us that then $(\delta, \delta)=\frac{10}{3}$ which satisfies (4.4). In Example 4, with $\pi_{1}(M)=Z_{5} \times Z_{5}$, choose

$$
\delta=\frac{1}{5}(-2,1,-1,2) .
$$


This again satisfies (4.4). Turning on the Wilson lines (4.6) and (4.7) in Examples 3 and 4 of $\S 3$, we find ourselves with two three generation models with gauge group $S U(3) \times$ $S U(2) \times U(1)$ at the string scale.

We have chosen to satisfy (4.4) separately after constructing $(0,2)$ models with $S U(5)$ gauge group in $\S 3$ only in order to make the physical interpretation (symmetry breaking by Wilson lines on a manifold $M$ with $\pi_{1}(M) \neq 0$ ) manifest. In general, one could orbifold by a $Z_{N}$ symmetry choosing any action on both the internal and the gauge degrees of freedom, as long as the combined system obeys the level-matching conditions.

\section{Conclusion}

In this paper we have discussed the construction of $(0,2)$ Calabi-Yau compactifications which can serve as suitable starting points for string model building. For $(2,2)$ string models, one has well developed techniques to compute the tree-level kinetic terms and the tree-level superpotential (which receives no string loop corrections). Similar progress in understanding the low energy effective actions of more general $(0,2)$ models is desirable. In addition, the data on unification of couplings in the minimal supersymmetric standard model provides a challenge for string models which yield $S U(3) \times S U(2) \times U(1)$ directly at the string scale (see e.g. [27] and references therein). The computation of threshold corrections in general $(0,2)$ models might help in addressing this concern.

\section{Acknowledgements}

I would like to thank J. Distler for very helpful comments on a draft and the authors of [6] for informing me of their results prior to publication. I would also like to thank Philip Candelas for correcting a bad choice of data in Example 4 in an earlier version of this paper. This work was supported in part by a fellowship from the Harvard Society of Fellows and by the William F. Milton Fund of Harvard University. 


\section{References}

[1] P. Candelas, G. Horowitz, A. Strominger, and E. Witten, "Vacuum Configurations for Superstrings," Nucl. Phys. B258 (1985) 46.

[2] E. Witten, "New Issues in Manifolds of SU(3) Holonomy," Nucl. Phys. B268 (1986) 79 .

[3] J. Distler and B. Greene, "Aspects of $(2,0)$ String Compactifications," Nucl. Phys. B304 (1988) 1.

[4] M. Dine, N. Seiberg, X.G. Wen and E. Witten, "Non-Perturbative Effects on the String World Sheet I," Nucl. Phys. B278 (1986) 769, "Non-Perturbative Effects on the String World Sheet II," Nucl. Phys. B289 (1987) 319.

[5] J. Distler and S. Kachru, "Singlet Couplings and (0,2) Models," Nucl. Phys. B430 (1994) 13, hep-th/9406090.

[6] E. Silverstein and E. Witten, to appear.

[7] J. Distler and S. Kachru, "(0,2) Landau-Ginzburg Theory," Nucl. Phys. B413 (1994) 213, hep-th/9309110.

[8] E. Witten, "Symmetry Breaking Patterns in Superstring Models," Nucl. Phys. B258 (1985) 75 .

[9] J.D. Breit, B. Ovrut, and G. Segre, " $E_{6}$ Symmetry Breaking in the Superstring Theory," Phys. Lett. 158B (1985) 33.

[10] B.R. Greene, "Special Points in Three-Generation Moduli Space," Phys. Rev. D40 (1989) 1645; B.R. Greene, K. Kirklin, P. Miron, and G.G. Ross, "A Three Generation Superstring Model II: Symmetry Breaking and the Low Energy Theory," Nucl. Phys. B292 (1987) 606, "A Three Generation Superstring Model I: Compactification and Discrete Symmetries," Nucl. Phys. B278 (1986) 667.

[11] R. Arnowitt and P. Nath, "Predictions from Three Generation Calabi-Yau String Theory," Phys. Rev. D42 (1990) 2948.

[12] C. Scheich and M. Schmidt, "Phenomenological Discussion of the Three Generation Gepner Model," Int. Jour. Mod. Phys. A7 (1992) 8021.

[13] D. Gepner, "String Theory on Calabi-Yau Manifolds: The Three Generations Case," Princeton preprint PUPT-88/0085.

[14] R. Schimmrigk, "A New Construction of a Three Generation Calabi-Yau Manifold," Phys. Lett. 193B (1987) 175.

[15] P. Candelas, M. Lynker, and R. Schimmrigk, "Calabi-Yau Manifolds in Weighted P4," Nucl. Phys. B341 (1990) 383.

[16] A. Klemm and R. Schimmrigk, "Landau-Ginzburg String Vacua," Nucl. Phys. B411 (1994) 559.

[17] See e.g. G. Aldazabal, A. Font, L. Ibanez, and A. Uranga, "String GUTS," hepth/9410206; A. Font, L. Ibanez, F. Quevedo, and A. Sierra, "The Construction of 
'Realistic' Four-Dimensional Strings through Orbifolds," Nucl. Phys. B331 (1990) 421 ; and references therein.

[18] See e.g. S. Chaudhuri, S. Chung, G. Hockney, and J. Lykken, "String Consistency for Unified Model Building," hep-ph/9501361; G. Cleaver, "GUTS with Adjoint Higgs Fields from Superstrings," hep-th/9409096; A. Faraggi, " $Z_{2} \times Z_{2}$ Orbifold Compactification - The Origin of Realistic Free-Fermionic Models," hep-ph/9501288; and references therein.

[19] T. Hubsch, Calabi-Yau Manifolds: A Bestiary for Physicists, World Scientific, 1992.

[20] M. Green, J. Schwarz, and E. Witten, "Superstring Theory: Volume 2," Cambridge University Press, 1987.

[21] P. Candelas, "Yukawa Couplings Between (2,1) Forms," Nucl. Phys. B298 (1988) 458.

[22] C. Vafa, "Modular Invariance and Discrete Torsion on Orbifolds," Nucl. Phys. B273 (1986) 592.

[23] J. Distler and S. Kachru, "Quantum Symmetries and Stringy Instantons," Phys. Lett. B336 (1994) 368, hep-th/9406091.

[24] G. Athanasiu, J. Atick, M. Dine, and W. Fischler, "Remarks on Wilson Lines, Modular Invariance, and Possible String Relics in Calabi-Yau Compactifications," Phys. Lett. B214 (1988) 55.

[25] S.P. DeAlwis, "Gauge Symmetry Breaking in Compactified Heterotic Strings," Phys. Lett. B216 (1989) 277.

[26] R. Slansky, "Group Theory for Unified Model Building," Phys. Rept. 79 (1981) 1.

[27] L. Ibanez, "Gauge Coupling Unification: Strings versus SUSY GUTs," Phys. Lett. B318 (1993) 73, hep-ph/9308365; L. Ibanez, D. Lust, and G. Ross, "Gauge Coupling Running in Minimal $S U(3) \times S U(2) \times U(1)$ Superstring Unification," Phys. Lett. B272 (1991) 251, hep-th/9109053. 